\title{
A Framework for Integrated Routing Protocols for Mobile Ad Hoc Network
}

\author{
Sherif M. Badr, PhD. \\ College of Computer science, \\ Modern Academy Cairo, Egypt
}

\begin{abstract}
A Mobile Ad hoc Network (MANET) is a collection of wireless mobile nodes that can dynamically form a network without using any pre-existing fixed infrastructure. Due to the mobility of the nodes, routing in mobile ad hoc network is not an easy task. A Number of mobile ad hoc routing protocols have been introduced. A set of performance comparison done for the most widely used ad hoc routing protocols; Destination Sequenced Distance Victor (DSDV), Dynamic Source Routing (DSR) and Ad Hoc On demand Distance Victor (AODV). The results of the simulation analysis which comes from these previous compressions show that DSR outperform the other two protocols in low load scenario but it has some weakness, and AODV outperform the other two protocols in high load scenario but also it has some weakness. This paper introduce a framework for new mobile Ad hoc routing protocol, Ad hoc Destination Sequenced Dynamic Source Routing (ADSDSR), which based on DSR mobile Ad hoc routing protocol and integrate some effective techniques from DSDV and AODV to improve the performance of the DSR. ASDSDR will be suitable in low and high load network.
\end{abstract}

Key word: wireless network, routing protocol, mobile network.

\section{INTRODUCTION}

Wireless network can be classified into infrastructure network which are a mobile nodes connected wirelessly but through a based station, where any node cannot connect directly to the other node, the connection done through the access point or the based station. The other type is the infrastructure which called a mobile ad hoc network, in this network each node connects to the other nodes wirelessly direct with any other infrastructure.

A Mobile Ad Hoc Network (MANET) consisting of wireless mobile nodes forming a temporary/short-lived network without any fixed infrastructure where all nodes are free to move join and disjoin where all nodes configure themselves. In MANET, each node acts both as a router and as a host at the time \& even the topology of network may also change rapidly as shown in fig 1 .

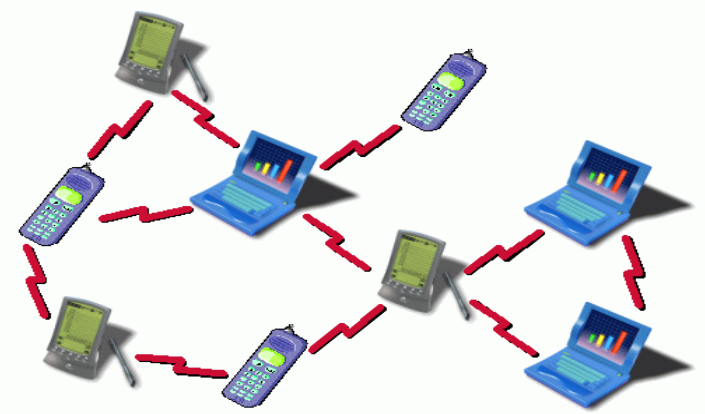

Fig 1: Mobile Ad Hoc Network

Mobile Ad Hoc network receive a great attention in last few years. MANET has many challenges, some of the challenges in MANET include:

1) Unicast routing

2) Multicast routing

3) Dynamic network topology

4) Speed

5) Frequency of updates or Network overhead

6) Scalability

7) Mobile agent based routing

8) Quality of Service

9) Energy efficient/Power aware routing

10) Secure routing

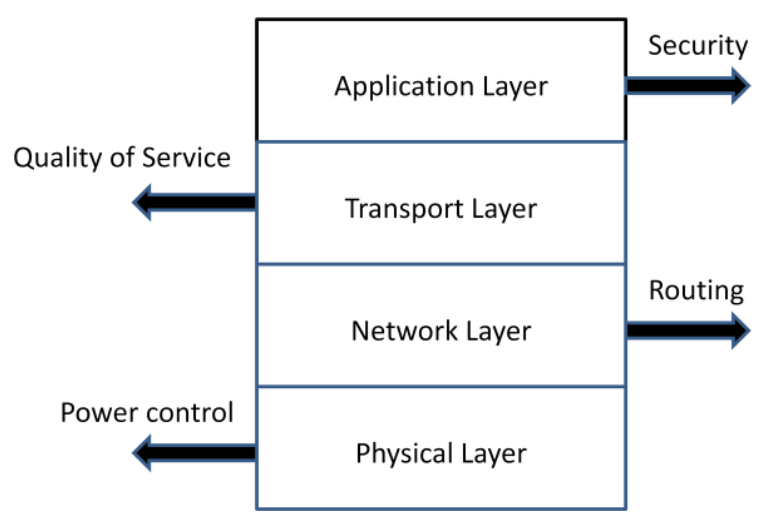

Fig 2 MANET Challenges

The key challenges faced at different layers of MANET are shown in Fig. 2. It represents layered structure and approach to ad hoc networks [2]. 


\section{MANET ROUTING PROTOCOLS}

Mobile ad hoc network characterized by the mobility of its nodes, each node can join and leave the network at any time; this means that the topology of the network also may changes at any time. These make the design of the mobile ad hoc network not an easy task ant it become one of the most important MANET challenges.

There are different criteria for designing and classifying routing Protocols for wireless ad-hoc networks. For Example, what routing information is exchanged; when and how the routing information is exchanged, when and how routes are computed etc.?[3]

Classification of routing protocols in MANET's can be done in many ways, but most of these are done depending on routing strategy and network structure. According to the routing strategy the routing protocols can be categorized as Table-driven and on demand (source initiated), while depending on the network structure these are classified as flat routing, hierarchical routing and geographic position assisted routing. Both the Table-driven and on demand protocols come under the Flat routing [11].

One of the most popular methods to distinguish mobile ad hoc network routing protocols is based on how routing information is acquired and maintained by mobile nodes. Using this method, mobile ad hoc network routing protocols can be divided, into proactive routing, also called a "table-driven" routing protocol, reactive routing, also called "on-demand" routing protocols, and hybrid routing. Hybrid routing protocols are proposed to combine the merits of both proactive and reactive routing protocols and overcome their shortcomings. Normally, hybrid routing protocols for mobile ad hoc networks exploit hierarchical network architectures. Fig 3 shows the classification of MANET routting protocols.

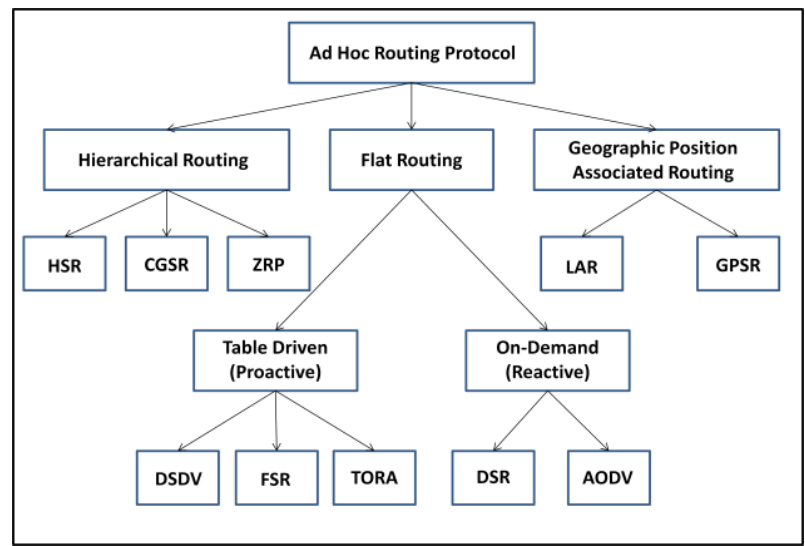

Fig 3 Classification of Routing Protocols in Mobile Ad hoc Networks

Compared to the proactive routing protocols for mobile ad hoc networks, less control overhead is a distinct advantage of the reactive routing protocols. Thus, reactive routing protocols have better scalability than proactive routing protocols in mobile ad hoc networks. However, when using reactive routing protocols, source nodes may suffer from long delays for route searching before they can forward data packets[12]

\subsection{Proactive routing protocol (Table-driven)}

In this group of protocols, each node maintains one or more tables that include routing information to other nodes of the network. All nodes update their tables to preserve compatibility and to give upgraded viewpoints of the network. When the topology of the network changes, the nodes distribute update messages across the network. Some identifying aspects of this class of routing protocols include the ways in which information is distributed, the ways the topology is changed and the number of tables necessary for routing.[13]. DSDV and TORA are proactive routing protocols.

\subsubsection{Destination-Sequenced Distance Vector $(D S D V)$ :}

DSDV is a hop-by-hop distance vector routing protocol each node must periodically broadcast routing updates. The advantage of DSDV over traditional distance vector protocols is that it guarantees loop-freedom. Each DSDV node maintains a routing table listing the "next hop" for each reachable destination. DSDV tags each route with a sequence number and considers a route $\mathrm{R} 1$ more favorable than $\mathrm{R} 2$ if $\mathrm{R} 2$ has a greater sequence number, or if the two routes have equal sequence numbers but $\mathrm{R}$ has a lower metric. Each node in the network advertises a monotonically increasing even sequence number for itself. When a node $\mathrm{B}$ decides that its route to a destination $\mathrm{D}$ has broken, it advertises the route to $\mathrm{D}$ with an infinite metric and a sequence number one greater than its sequence number for the route that has broken (making an odd sequence number). This causes any node A routing packets through $\mathrm{B}$ to incorporate the infinite-metric route into its routing table until node $\mathrm{A}$ hears a route to $\mathrm{D}$ with a higher sequence number as shown in fig 4 [5].

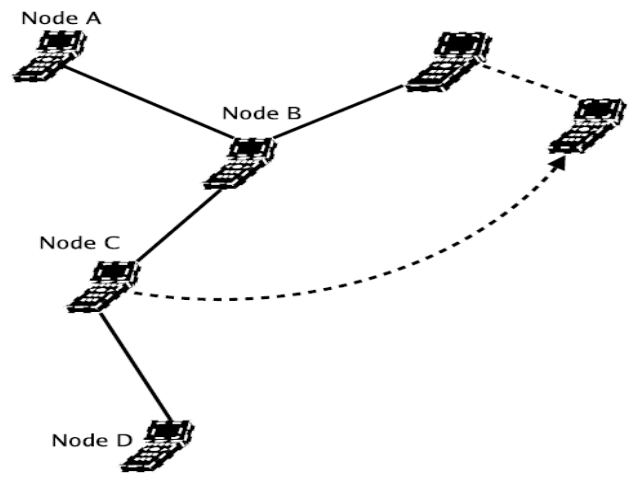

Fig 4 Broken links affecting the network routing

\subsubsection{Temporary Ordered Routing Algorithm TORA}

TORA (Chaudhry, 2005) is a source-initiated on demand routing protocol, which uses a link reversal algorithm and provides loop-free multi-path routes to a destination node. In TORA, each node maintains its one-hop local topology information and also has the capability to detect partitions. TORA is proposed to operate in a highly dynamic mobile networking environment. The key design concept of TORA is the location of control messages to a very small set of nodes 
near the occurrence of a topological change. The protocol performs three basic functions (a) route creation (b) route maintenance (c) route erasure.[8]

\subsection{On-Demand Protocols}

In comparison with table-driven routing protocols, all updated routes are not maintained in each node in this group of protocols; instead, routes are constructed only when it is necessary. When an origin node wants to send something to a destination, it makes are quest to the destination for the route detection mechanisms. For this reason, this type of protocol is known as a reactive protocol. This route remains valid until the destination is accessible [13]. DSR and AODV is reactive routing protocols.

\subsubsection{The Ad hoc On-Demand Distance Vector (AODV)}

AODV routing protocol uses mobile nodes to identify routes faster to reach new destinations and does not require nodes to maintain routes to destinations which are not in active communication. The AODV algorithm enables dynamic, autonomous, multi hop routing between mobile nodes to establish and maintain an ad hoc network.

AODV responds to route breakages and changes in network topology in a timely manner. The operation of AODV is loopfree and avoids the Bellman-Ford "counting to infinity" problem offers fast convergence when the ad hoc network topology changes as shown in fig 5. When links break, AODV causes the affected set of nodes to be notified so that they are able to invalidate the routes using the lost link Ever since the first demand driven routing protocol various modifications have been made in the basic algorithm for the better utilization of ad hoc networking [7].

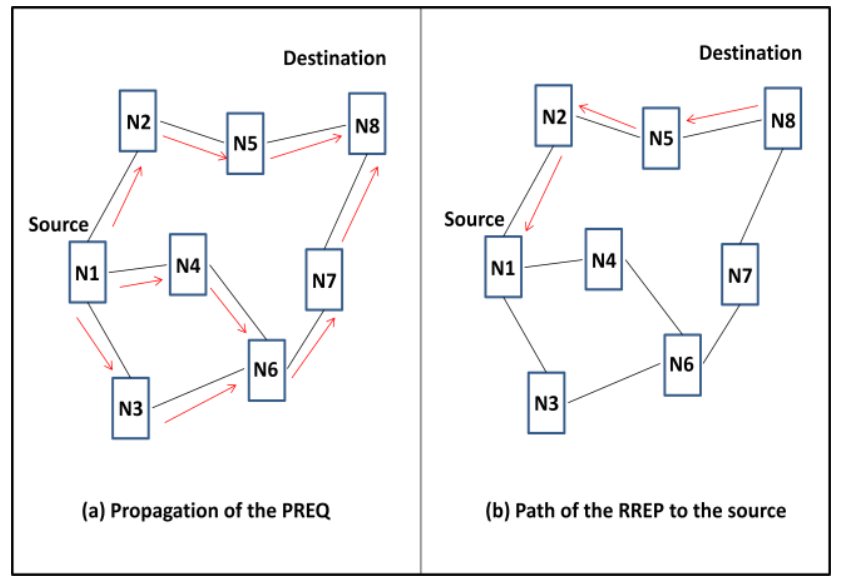

Fig 5: packet delivery nodes in AODV

\subsubsection{Dynamic Source Routing (DSR)}

Dynamic Source Routing is a reactive routing protocol which uses source routing to deliver data packets. Headers of data packets carry the sequence of nodes through which the packet must pass. This means that intermediate nodes only need to keep track of their immediate neighbors in order to forward data packets. The source, on the other hand, needs to know the complete hop sequence to the destination. As in AODV, the route acquisition procedure in DSR requests a route by flooding a Route Request packet. A node receiving a Route Request packet searches its route cache, where all its known routes are stored, for a route to the requested destination. If no route is found, it forwards the Route Request packet further on after having added its own address to the hop sequence stored in the Route Request packet. The Route Request packet propagates through the network until it reaches either the destination or a node with a route to the destination. If a route is found, a Route Reply packet containing the proper hop sequence for reaching the destination is unicasted back to the source node [3].

DSR does not rely on bi-directional links since the Route Reply packet is sent to the source node either according to a route already stored in the route cache of the replying node, or by being piggybacked on a Route Request packet for the source node. However, bi-directional links are assumed throughout this study as shown in fig 6 .

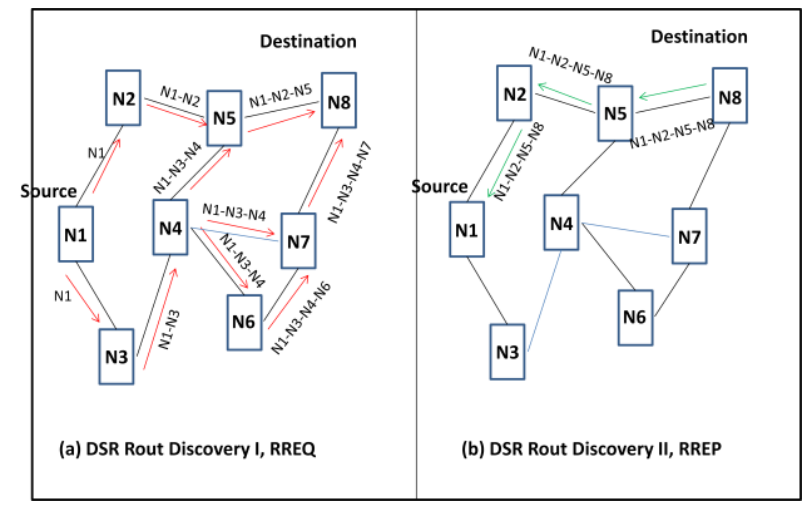

Fig 6: DSR Route Discovery.

Then the reverse path in the Route Request packet can be used by the Route Reply message. The DSR protocol has the advantage of being able to learn routes from the source routes in received packets. To avoid unnecessarily flooding the network with Route Request messages, the route acquisition procedure first queries the neighboring nodes to see if a route is available in the immediate neighborhood. This is done by sending a first Route Request message with the hop limit set to zero, thus it will not be forwarded by the neighbors. If no response is obtained by this initial request, a new Route Request message is flooded over the entire network [3].

\section{COMPARISON OF MANET ROUTING PROTOCOLS}

A comparison between the three routing protocols DSR, AODV and DSDV is done in [4] using ns 2 simulator. When node mobility and node density increase in the network, DSR routing protocol performance is quite well compared to AODV and DSDV under condition of keeping source node fixed and destination node variation. Also while keeping the destination node fixed and source node variation, DSR performance improves much better compared to AODV as well as DSDV routing so, DSR performs efficient for the network, the weakness would be much in DSDV routing protocol. Finally, when the node density increases then DSDV performance 
more poor and it goes nearly to zero value. Also, here the performance of DSR routing protocol is much better than AODV and DSDV. So, under high traffic condition DSR performs well and is good for engineers while designing any ad-hoc real scenario network.[4]

Table 1 shows the Simulation Parameters for varying network [6] where mobility of the nodes kept a constant for varying network size with constant mobility to analyze the performance of AODV, DSR, DYMO, OLSR and ZRP: The analysis showed on demand routing protocols like DSR and DYMO shows good stable results of throughput on varying node density.

Table 1 Simulation Parameters for varying network

\begin{tabular}{|c|c|}
\hline parameters & Values \\
\hline simulator & qualNet \\
\hline Protocols Studied & AODV,DSR,DYMO,OLSR\&ZAP \\
\hline Number of nodes & $20,50,100,200$ nodes \\
\hline Simulation time & 100 s \\
\hline Simulation area & 700*700 sq $\mathbf{m}$ \\
\hline Node movement model & Random waypoint mobility \\
\hline Traffic types & Values \\
\hline Mobility of nodes & Min speed=1m/s,Max speed=10m/s \\
\hline Rate of packet generation & 20 packets $/ \mathrm{s}$ \\
\hline Size of packets & 1000 bytes \\
\hline
\end{tabular}

AODV throughput decreases for high density networks. DSR has an edge over other protocols in successfully delivering data packets for varying node density. DYMO and AODV are close behind. DSR is better in transmission of packets per unit time and maximum number of packets reached their destination successfully with some delays. Whereas AODV \& DYMO having almost same values in all of the performance metrics, they transmit packets with very less delay but transmits less packets to their destination as compare to DSR.[6]

For high node density OLSR shows highest jitter and, ZRP and DSR are next to it. Average Jitter of DSR is high for varying network. End to end delay is more in ZRP as compared to others as ZRP operation of route discovery uses additional time as it uses IARP, IERP and BRP leading to more number of control packets. OLSR is next trailed to ZRP and DSR is behind OLSR as it uses source routing. Waiting time for packets in DSR is long as it builds long queue is attributed to use of aggressive caching which increases with increase in network size. Next highest waiting time is for ZRP and OLSR packets as observed for denser networks [6].

Table 2 shows best protocols for 20, 30, 75 nodes after simulation results.
Table 2 - Best Protocols Individually

\begin{tabular}{|c|c|c|c|}
\hline Parameters & 20 nodes & 30 nodes & 75 nodes \\
\hline Npkts & DRS & DRS & TORA \\
\hline Nlost & DRS & AODV & DRS \\
\hline Bytes & TORA & DRS & TORA \\
\hline Bitrate & TORA & DRS & TORA \\
\hline Bitra & TORA & DRS & TORA \\
\hline Last PK Time & DRS & DRS & TORA \\
\hline Packet loss rate & DRS & AODV & DRS \\
\hline Packet delay & DRS, TORA & DRS, TORA & DRS, TORA \\
\hline
\end{tabular}

In [8] present the simulation of NS 2 as well as compared the performance of DSDV, AODV and DSR routing protocols by taking 6 parameters versus time. DSR performs best in the simulation results. DSR performs well for 20 nodes as well as 30 nodes for almost all parameters .DSR shows worst performance in case of packet lost by using 30 nodes. For 75 nodes TORA performs well as compared to DSR. AODV performs better in case of packet lost by using 30 nodes. And for remaining cases AODV neither performs best nor worst. DSDV performs worst for almost all cases when we use 20 nodes as well as 30 nodes \& 75 nodes in our simulation. TORA performs best in 11 out of 24 cases. With 75 nodes TORA performs well. So we can say that with large network TORA performs well.

In [9], two routing protocols named DSDV and AODV are simulated and compared under specific scenarios with WSNs environment. With the help of the NS2 simulator program, DSDV and AODV are evaluated in respect of packet delivery fraction, end to end delay and average throughput. However, the simulation results reveal that there is no one protocol which is better than the other. Each protocol has its own advantages as well as its disadvantages making it suitable for some applications and not for others. Hence, an efficient routing protocol should be selected that suits the desired sensing task.

Under packet delivery fraction, AODV has better performance than DSDV in the considered scenarios. PDF increases with an increase in the pause time for both protocols. As far as throughput is concerned, AODV performs by far better compared to DSDV. Average throughput in both protocols decreases steadily with an increase in the number of expired nodes and in case of pause time, the average throughput increases with increasing pause time.

AODV suffers from delay. Regardless of the period of pause time, it shows longer average end-to end delay in comparison with DSDV in the considered scenarios. However, the time delay fluctuates in DSDV protocol with an increase in pause time. On the other hand, end-to-end delay increases in AODV with increasing pause time.

\subsection{Critiques of DSDV}

DSDV requires nodes to periodically transmit routing table updates packets regardless of the network traffic. When the number of nodes in the network grows the size of the routing tables and the bandwidth required to update them also grows. 
This overhead is considered as the main weakness of DSDV. DSDV also pose a period of convergence before which routes will not be known and packets will be dropped. This could also limit the number of nodes that can connect to the network since the overhead grows as $\mathrm{O}\left(\mathrm{N}^{\wedge} 2\right)$. Moreover, DSDV works only with bidirectional links. In addition, in DSDV routing loops can occur while the network is reacting to a change in the topology.[10]

DSDV use distance vector shortest-path routing as the underlying routing protocol. It has a high degree of complexity especially during link failure and additions. Maximum settling time is difficult to determine in DSDV. DSDV does not support multi-path routing. Fluctuation is another problem of DSDV. In some simulation studies, DSDV is much more conservative in terms of routing overhead but because link breakages are not detected quickly more data packets are dropped. Specification of DSDV is silent over security issue . DSDV assumes that all nodes are trust worthy and cooperative. Once the false sequence has been established the attacker will continuously send out new packets to update the value. Therefore more hosts will be cheated as a single misbehaving node can pose a serious threat for the entire network [10].

\subsubsection{DSDV Advantages:}

The main contribution of the algorithm was to solve the Routing Loop problem. Each entry in the routing table contains a sequence number, the sequence numbers are generally even if a link is present; else, an odd number is used. The number is generated by the destination, and the emitter needs to send out the next update with this number [14].

\subsubsection{DSDV Disadvantages}

DSDV requires a regular update of its routing tables, which uses up battery power and a small amount of bandwidth even when the network is idle. DSDV is not suitable for highly dynamic networks. (As in all distance vector protocols, this does not perturb traffic in regions of the network that are not concerned by the topology change.)[14]

\subsection{Critiques AODV}

AODV is an on demand approach but still use periodic broadcast of „hello message ee to track neighboring nodes. This periodic propagation causes network overhead in AODV. In AODV a route has to discover prior to the actual data packet transmission. This initial search latency may degrade the performance of interactive applications. Similarly the quality of path is not known prior to call set-up. It can be discovered only while setting up the path. Moreover quality of path must be monitored by all intermediate nodes in an active session at the cost of additional latency and overhead penalty .That makes AODV quite unsuitable for real life applications. AODV cannot utilize routes with asymmetric links between nodes and thus require symmetric links . Nodes in AODV store only route that are needed. Nodes use the routing caches to reply to route queries. These results in „uncontrolled replies and repetitive updates in hosts ${ }^{\text {ee }}$ caches yet early queries cannot stop the propagation of all query messages which are flooded all over the network [10].

\subsubsection{AODV Advantages}

The advantage of AODV is that it tries to reduce the number of required broadcasts. It creates the routes on an on demand basis, as opposed to maintain a complete list of routes for each destination [15].

An important feature of AODV is the maintenance of timerbased states in each node, regarding utilization of individual routing table entries. A routing table entry is said to be expired if not used within certain duration. These nodes are notified with route error (RERR) packets when the next-hop link breaks. In the situation of link break, each predecessor node, forwards the RERR to its own set of predecessors. In this way all routes, which contain the broken link, are removed [16].

This strategy reduces the effects of stale routes as well as the need for route maintenance for unused routes. Another distinguishing feature of AODV is the ability to provide unicast, multicast and broadcast communication. AODV uses a broadcast route discovery algorithm and then the unicast route reply massage [17]. AODV is designed for up to thousands of nodes. In general AODV performed better in dense environment [18].

\subsection{AODV Disadvantages}

Other protocols generally outperform AODV in less stressful situations, so it not suitable in small networks less number of nodes and less mobility [17].

\subsection{Critiques of DSR}

DSR is not designed to track topology changes occurring at a high rate. Two sources of bandwidth overhead in DSR are route discovery and route maintenance. These occur when new routes need to be discovered or when the network topology changes. In DSR this overhead can be reduced by employing intelligent caching techniques in each node at the expense of memory and CPU resources. The remaining source of bandwidth overhead is the required source route header included in every packet. This overhead cannot be reduced by techniques outlined in the protocol specification.[10]

DSR is based on source routing thus requires considerably greater routing information. In DSR a route has to discover prior to the actual data packet transmission. This initial search latency may degrade the performance of interactive applications. Moreover, the quality of path is not known prior to call setup. It can be discovered only while setting up the path. This quality of path needs monitoring by all intermediate nodes during a session. It increases the cost of additional latency and overhead penalty [10].

Due to source routing DSR has major scalability problem. Nodes use routing caches to reply to route queries. This results in an uncontrolled. replies and repetitive updates in hosts caches. In addition, early queries cannot stop the propagation of all query messages which are flooded all over the network. Therefore when the network becomes larger, the control packets and message packets also become larger. This could 
degrade the protocol performance after a certain amount of time.[10]

\subsubsection{DSR Advantages:}

The key advantage of source routing is that intermediate nodes do not need to maintain up-to-date routing information in order to route the packets they forward, since the packets themselves already contain all the routing decisions [19].

DSR uses no periodic routing messages, thereby reduces network bandwidth overhead, conserves battery power and avoids large routing updates [20].

\subsubsection{DSR Disadvantages:}

Certain features of DSR hurt its performance or make it vulnerable to security attacks. DSR has no Expiration of Routes. DSR Without an effective mechanism to remove excessively old (stale) entries, route caches may contain broken or non-minimum hop routes. Using stale routes causes loss of data packets (low delivery rate) and wastes network bandwidth. Route replies from intermediate nodes and snooping data packets exacerbate this problem by polluting caches with stale routes [21].

\subsection{Critiques of TORA}

TORA is one of the largest protocol thus requires extra memory for different operations. Each node must maintain a structure describing the nodes height as well as the status of all connected links per connection supported by the network. TORA requires each node to be in constant coordination with neighboring nodes, to detect topology changes and coverage which pose high bandwidth and CPU requirements. The main drawback of TORA is the exorbitant assumptions that it makes. Not only does it require bi-directional links and a linklevel protocol but it actually depends on correct and in-order transmission of all packets. TORA uses intermodal coordination and it exhibits instability behavior similar to "count-to-infinity" problem in distance vector routing protocols. Thus there is a potential for oscillations to occur especially when multiple sets of coordinating nodes are concurrently detecting partitions, erasing routes, and building new routes based on each other. Though such oscillations are temporary and route convergence will ultimately occur, it poses real threat to utilize TORA at its full [10].

\section{THE PROPOSED ROUTING PROTOCOL: AD HOC DESTINATION-SEQUENCED DYNAMIC SOURCE ROUTING (ADSDSR)}

This research tries to reach to an effective mobile Ad hoc routing protocol. Many research reached that DSR is more suitable Ad Hoc routing protocol in small and low load (speedlnode) network, but it needs some improvement.

When taking the DSDV, DSR and AODV Ad Hoc routing protocols as a basic and most widely used routing protocols in mobile Ad Hoc networks and also as they are so closed to each other in their techniques, it founds some facts as; DSR, consistently generates less routing load than AODV and DSDV. However, the poor performances of DSR are mainly attributed to aggressive use of caching, and lack of any mechanism to expire stale routes or determine the freshness of routes when multiple choices are available. Aggressive caching seems to help DSR at low loads and keeps its routing load down.

This means that DSR Ad Hoc routing protocol need two mechanisms to be improved the first is a mechanism to determine the freshness of the route when multiple choices are available and the second is a mechanism to expire stale route to be more effective. To solve the first problem of missing a mechanism to determining the freshness of the route, the mechanism of destination sequence number which used in the DSDV routing protocol will be used but after some modification.

Moreover, to solve the second problem of missing a mechanism to expire stale route, the mechanism of routing table expiration timer that used in AODV routing protocol will be used but also after some modification, and use a timer to delete the unused routes in the cash. Also trying to change the technique of route selected in DSR, to reach better end to end delay.

\subsection{Description of Ad Hoc Destination Sequenced Dynamic Source Routing Protocol (ADSDSR):}

ADSDSR is an integrated version of DSDV, DSR and AODV Ad Hoc routing protocol. ADSDSR protocol is like DSR protocol it is an on demand routing protocol this means that route is established on demand of the source or when the source need. It uses the source routing, which is the key distinguishing feature of DSR, it means that the source knows the complete hop by hop route to the destination stored in route cache which means that each node has its own route cache.

ADSDSR can maintain multiple route cache entries for each destination. The data packets carries the source route in the packet header and the intermediate nodes do not need to spend any resource or energy to maintain a routing table for each data packet. There is also no need for periodic routing advertisement messages, which will lead to reduce network bandwidth overhead, and save the battery power for the mobile hosts.

ADSDSR has two main components as in DSR: route discovery and route maintenance. Route discovery is used by nodes for discovering new routes when they do not have routes to destinations they need to communicate with in their cache. Route maintenance is used for discovering routes that have been broken due to node movements or failures. When a route is broken, either a different existing (less optimal) route may be used or the node may initiate route discovery for discovering a new route. Now, each of these two components of ADSDSR will be discuss in more detail. 


\subsubsection{Route Discovery Process of ADSDSR:}

As in DSR when the source node needs to send data to the destination it check its cache first for the route. If there is a route to the destination, the source node will use it, else it will initiates the route discovery process.

However, ADSDSR uses the sequence number, which used in DSDV, it means that each node has sequence number, this sequence number incremented after any movement of this node. Each route ended by the destination sequence number to determine the freshness of the route.

If there is no route to the destination, the source initiate the route discovery process by broadcasting a route request message RREQ. The RREQ message contains the address of the source and destination (similar to AODV). The RREQ also has a unique identity associated with it. When a neighbor's node receives the RREQ, it checks if it sees the combination of the source node and the unique identity or not. If the node has seen this request before, it just ignores it. Else, if it has not seen this combination before, it check if this node is the destination it will send back a route reply message (RREP) to the source that contains the route to the target given by the node list and put the destination sequence number at the end of the route. if it is not a destination it check if it has a route to the destination in its cache, if so it will append its own cached route with the node list of the RREQ then the node will send a RREP to the source with the route to the target. If it is not the destination and did not have a route to the destination, it appends its address in the RREQ and then rebroadcasts the message to its neighbors.

The source will receives all the RREPs, and check first the destination sequence number for each route to take the most recent destination sequence number, and neglect the routes with the older destination sequence number. If there are two RREPs that have routes with the same destination sequence number, it will take the route which comes from the first RREP reached to the source (coming from AODV but after some manipulation), and sort the rest routes according to their reaching time, first reached first taken,( FIFO: first in first out). This automatically favors the least congested route instead of the shortest route because that the shortest route not always the best route. If there are two RREPs reached at the same time and with the same destination sequence number, the source has to take the one with smallest hop number.

A proposed conceptual framework for route discovery process of the new mobile Ad hoc routing protocol ADSDSR designed. Fig. 7 show the Pseudo code which tries to simplify the process of route discovery process of mobile Ad hoc routing protocol ADSDSR.

\section{Pseudo code:}

Step 1: Initialize source equal to $S$.

Step 2: $S$ needs to send data to packets to destination D.

Step 3: initialize Destination equal to D

Step 4: if the cache of $S$ has a route of $D$ then use this route to send data packets to $D$ and then Terminate Else go to step 5
Step 5: initiate route discovery process

Step 6: Set PREQ ID and Put the address of $S$ and D in PREQ

Step 7: Broadcast PREQ message to all neighbors.

Step 8: neighbor nodes receive PREQ message

Step 9: if it see the combination of the source node $S$ and PREQ $I D$ before the PREQ is ignored and current node go nothing then go to step 8 else go to step 10

Step 10: if this node is the target $D$ then send PREP message to $S$ containing the route to $D$ given by the node list ended by destination sequence number and go to step 13 else go to step 11.

Step 11: if it has a route to $D$ in its cache then send PREP message to $S$ and concatenate the node list in the PREQ with its own cached route to $D$ ended by the destination sequence number and go to step 13 else go to step 12.

Step 12: the node adds its own address to the node list of PREQ and rebroadcast PREQ message to the neighbors.

Step 13: $S$ receives PREP messages.

Step 14: if the destination sequence number for each route is the recent one then take the route which comes from the first $P R E P$ reached to $S$ Else neglect the route and go to step 13.

Step 15: if there are two or more PREP reached at the same time then take the route with the smallest hop number and start to send data packets to D else go to step 16

Step 16: start to send data packets to D

Step 17: try to sort the rest of routes according to their reached time, then to the smallest hop count to be ready for the next transmission between $S$ and $D$ if needed then terminate the process.

\section{Fig 7 the Proposed Conceptual Framework for Route Discovery Process of ADSDSR}

\subsubsection{Route Maintenance Process of ADSDSR:}

If any node detect that the packet cannot be transmitted to the next hop, the node has to send a route error message (RERR) to the source node of the packet that could not be delivered (as in DSR). This RERR identifies the link over which the packet could not be forwarded. When the source receives the RERR, it will delete all routes containing this link from its cache. In addition, the node has to broadcast RERR to all nodes in the network (as in AODV) to let them delete the broken link from their cache. If the source wants to send packet to the same destination, it will check its cache first and take the first route in the sorted route cache, if there is no any route to this destination, the source node needs to initiate the route discovery process again.

If there is a route in the node's cache has still did not used for a long period of time, this route has to be expired and deleted from the cache( as in AODV) to ensure the freshness of the routes in each node's cache. The expiration time must be determined according to the rate of sending message of the node and the speed of the node in the network.

A proposed conceptual framework for route maintenance process of the new mobile Ad hoc routing protocol ADSDSR designed in flowchart form, fig 8 shows this flowchart which 
tries to simplifies the process of route maintenance process of mobile Ad hoc routing protocol ADSDSR.

\subsection{Ad Hoc Destination Sequenced Dynamic Source Routing (ADSDSR) Contribution and Its Effects}

ADSDSR is like DSR, it is on demand routing protocol that did not use any periodic advertising, this lead to less routing load and overhead than other protocol like the proactive routing protocols. It has the faster route discovery process because it allows multiple routes to one destination in their cache.

ADSDSR produce a new contribution summarized in four points:

1- The first contribution comes from ADSDSR is that it solves the problem of missing a mechanism of keeping the freshness of the routes in DSR. It use the technique of destination sequence number (as in DSDV) to ensure that the selected route is a fresh route (the newest one), by keeping and select the route with the up-to-date destination sequence number and neglect all route with the old destination sequence number. This technique tries to solve the problem of stale route. The stale route problem comes from when the source node needs to send a package to any destination, it check its cache first, if the cache not fresh enough and not updated the source node will use a stale route which may have any broken links, this means that the packet cannot be send to the destination, which lead to package loss and wasting time in another route discovery process. Therefore, if the cache has fresh routes this means that all routes in the node cache are active routes. Moreover, if there is no route to the destination, the source will start a route discovery process, which will save time than use a stale route and loss package, and then start a route discovery process again. This means less packet loss, high throughput and high delivery ratio. In addition, this technique always let the cache free enough for new active and fresh routes, this means that it keep a free space in the route cache.

\section{Pseudo Code:}

Step 1: the node detect that the packet cannot be transmitted to the next hop.

Step2: the node send PERR message to $S$ that the packet could not be delivered

Step 3: the node send PERR to all nodes in the network that identifies the link aver which has the error (which the packet could not be forwarded)

Step 4: all the routes containing the link will be deleted from all nodes in the network including $S$

Step 5: if $S$ needs to send packet to the same destination then start route discovery process of ADSDSR Else Go to step 6

Step 6: Terminate the process.

Fig. 8 the Proposed Conceptual Framework for Route Maintenance Process of ADSDSR

2- The second contribution is it uses the technique of route selection from AODV but after some manipulation. The source node selects the route with the most resent destination sequence number. If there are two or more routes have the same destination sequence number the source will sort them according the arriving time (first reached first taken) This automatically favors the least congested route instead of the shortest route because that the shortest route not always the best route. In addition, if there are two or more routes with same destination sequence number also reached to the source at the time then the route with the less hop number will be taken. This technique will improve the delay of the ADSDSR protocol.

3- The third contribution comes with ADSDSR is the route expiration time (as in AODV) which expire any route if it did not use for a long time. Each route in the cache has an expiration time; if it did not used within this period of time the route will be expired and automatically deleted from the cache. This technique will help in solving the problem of use stale route as discussed before. In addition, it helps the cache to have a free space to accept a new active route.

4- The forth contribution comes with ADSDSR, when any node detect that it cannot send a packet to it neighbor this means that there exist a broken link, it will send RERR to the source to delete all the route which containing the broken link in its cache (as in DSR). In addition, it broadcast RERR to all nodes in the network (as in AODV) to ensure that this broken link deleted from the entire network, to solve the problem of existing of the stale routes in the network.

\section{CONCLUSION}

Mobile Ad hoc network (MANET) is an important area in wireless network and gain a great attention between other areas. MANET has many challenges, routing protocols is one of an important one of them due to the mobility of the nodes and the periodically changes in the topology.[22]

Many routing protocols for MANET have been introduced, and as said before there are some protocols are most commonly used and they prove there efficiency from the performance comparison which done for them. From the most commonly used protocols there are three protocols are so closed to each other, these protocols are DSDV as proactive routing protocol, DSR and AODV as reactive routing protocols. They perform well in some performance metrics while there are some significant weaknesses in other performance metric.

The results of these comparisons show that the reactive routing protocol DSR and AODV outperform the other type the proactive routing protocols DSDV and TORA in many scenarios, this is due to the reactive routing protocols don't exhaust the network by many notification and passing messages so that they not overloud the network, they send the message or the request on demand not periodically.

On the other hand the proactive routing protocol exhaust the network by periodic request and notification which overloud the network which effect the delay by increasing the delay that's make the reactive routing protocols more preferable which have less delay. 
However when comparing the two reactive routing protocols, the DSR and the AODV founded that the DSR MANET routing protocol outperform AODV in low loud scenarios(less nodes and less mobility) but AODV outperform the DSR in high loud scenarios( large number of nodes and high mobility).

When talking about the reactive MANET routing protocols, found that AODV is designed for up to thousands of nodes while DSR is designed up to two hundred nodes. AODV performed better in dense environment or high load scenarios (big number of nodes andlor high mobility). DSR performed better in less "stressful" situations, (smaller number of nodes and/or lower mobility). AODV and DSR are proved to be better than DSDV. While it is not very clear that any one protocol is best for all the scenarios, each protocol is having its own advantages and disadvantages and may be well suited for certain scenarios.

Even though DSR and AODV share the on-demand behavior, much of their routing mechanics are different. In particular, DSR uses source routing and route caches and does not depend on any periodic or timer-based activities. DSR exploits caching aggressively and maintains multiple routes per destination. AODV, on the other hand, uses routing tables, one route per destination, and destination sequence numbers, a mechanism to prevent loops and to determine freshness of routes which come from DSDV routing protocol. The general observation from the comparison is that DSR outperforms AODV in less "stressful" situations, i.e., smaller number of nodes and lower load and/or mobility. AODV, however, outperforms DSR in more stressful situations (e.g., more load, higher mobility).

DSR needs any mechanism to expire the stale routes or to determine the freshness of routes when multiple choices are available. Aggressive caching, however, seems to help DSR at low loads and also keeps its routing load down. A mechanism to expire routes and/or determine freshness of routes will benefit DSR's performance significantly. On the other hand, AODV's routing loads can be reduced considerably by source routing the request and reply packets in the route discovery process.

All of the above lead to the need for new mobile ad hoc routing protocol which tries to solve the weakness of the other protocols. ADSDSR is a new proposed Ad hoc routing protocol which tries to integrate the three most widely used ad hoc routing protocols, DSDV, DSR, AODV and reached to a new effective one.

ADSDSR tries to solve the DSR problems by integrating some feature from DSDV and AODV to DSR but after some modification, this integration and modifications gives ADSDSR the power to be more effective than the other ad hoc routing protocols. ADSDSR is an improved version from DSR, but it will be suitable not only in low load scenarios but also in high load scenarios.

The research conclusion can be summarized in the following points:

1 -The research produces a new ad hoc routing protocol ADSDSR that can be suitable in low and high load scenarios.
2- ADSDSR is an improved version from DSR, so it takes the advantages of source routing and on demand routing protocol from DSR which will be more suitable for mobile Ad hoc network than other types of routing protocols.

3- ADSDSR integrates the destination sequence number from DSDV to ensure that the routes in ADSDSR are fresh enough and avoid the stale route problem. This will decrease the packet loss, delay and increase the delivery ratio and throughput.

4- ADSDSR integrates the route selection from AODV which select the first reached route comes from RREP, which means that it, select the least congested route rather than select the shortest bath. This will decrease the delay.

5- ADSDSR integrates the table expiration time from AODV; it uses cache expiration time to delete unused routes within this time. This also ensures the freshness of routes and avoids the stale route problem. This will free the route cache for new fresh route, decrease the delay, packet loss and increase the packet delivery ratio.

6- ADSDSR integrates the route maintenance from AODV to let all nodes in the network to know the broken link not the source only. This also helps in removing stale routes and keeps the freshness of the routes.

\section{REFRENCES}

[1] B.Soujanya et al.,"Study of Routing Protocols in Mobile Ad-hoc Networks", International Journal of Engineering Science and Technology (IJEST), ISSN: 0975-5462, Vol. 3 No. 4, April 2011.

[2] Sunil Taneja and AshwaniKush , "A Survey of Routing Protocols in Mobile Ad Hoc Networks", International Journal of Innovation Management and Technology, ISSN: 2010-0248, Vol. 1, No. 3, August 2010.

[3] V.B.NARSIMHA, "Comparison of Routing Protocols in Mobile Ad Hoc Networks", Global Journal of Advanced Engineering Technologies, ISSN: 2277-6370, Vol1, Issue1, 2012.

[4] Shrikant Upadhyayet al," Comparison and performance analysis of reactive type DSR, AODV and proactive type DSDV routing protocol for wireless mobile ad-hoc network, using NS-2 simulator", Journal of Engineering and Computer Innovations, ISSN 2141-6508, Vol. 2(10), pp. 36-47, March 2012.

[5] M.S.R.S PRASAD et al. ," A Comparison Study Of DSDV And SEAD Wireless Ad Hoc Network Routing Protocols", [IJESAT] International Journal Of Engineering Science \& Advanced Technology, ISSN: 2250-3676,Volume - 2, Issue - 1,pp. 59 - 63, Jan-Feb 2012.

[6] Dr. Ritika\& Dr. Nipur, "Performance Evaluation of Reactive, Proactive and Hybrid Routing Protocols Based on Network Size for MANET", International Journal of Computer Science and Security (IJCSS), Volume (6), Issue (1) , 2012 .

[7] G. Rajkumar, dr. K. Duraisamy, "A Review Of Ad Hoc OnDemand Distance Vector Routing Protocol For Mobile Ad Hoc Networks", Journal of Theoretical and Applied Information Technology, ISSN: 1992-8645,Vol. 36, No.1, 15th February 2012. 
[8] Er. Saurabh Mittal, "Performance Evaluation Of AODV, DSR, DSDV and TORA Routing Protocols" , International Journal of Multidisciplinary Research, ISSN 2231 5780, Vol.2 Issue 2, February 2012,

[9] Adel.S.Elashheb,"Performance Evaluation of AODV and DSDV Routing Protocol in Wireless Sensor Network Environment", International Conference on Computer Networks and Communication Systems (CNCS),vol.35,Singapore, 2012.

[10] HumayunBakht, "Survey of Routing Protocols for Mobile Ad-hoc Network",International Journal of Information and Communication Technology Research, Volume 1 No. 6, ISSN-2223-4985, October 2011

[11] JyotsnaRathee, Simulation, "Analysis and Comparison of DSDV Protocol in MANETS ", degree of Master of Engineering in Computer Science \& Engineering, Computer Science And Engineering Department, Thapar University, Patiala, MAY 2009

[12] Subir Kumar Sarkar, T G Basavaraju, C Puttamadappa,"Ad Hoc Mobile Wireless Networks Principles, Protocols, and Applications", Auerbach Publications,Taylor \& Francis Group, (C) 2008

[13] Ali Norouzi and A. HalimZaim, "Energy Consumption Analysis of RoutingProtocols in Mobile Ad Hoc Networks", Machine Intelligence Research LabsDepartment of Computer Engineering, Istanbul University,Avcilar, Istanbul,Turkey,2012.

[14] Abdul Hadi Abd Rahman, Zuriati Ahmad Zukarnain, "Performance Comparison of AODV, DSDV and I-DSDV Routing Protocols in Mobile Ad Hoc Networks" . European Journal of Scientific Research, ISSN 1450-216X Vol.31 No.4, pp.566-576, EuroJournals Publishing, Inc. http://www.eurojournals.com/ejsr.htm, 2009.

[15] Vineet Agrawal , Dr Yashpal Singh,Manish Varshney ,Vidushi Gupta, "Routing protocols in wireless networks", (IJCNS) International Journal of Computer and Network Security, Vol. 2, No. 4, India, April 2010.
[16] N Vetrivelan, Dr. A V Reddy, "Performance Analysis of Three Routing Protocols for Varying MANET Size", Proceedings of the International Multi Conference of Engineers and Computer Scientists, Vol II, Hong Kong, 1921 March, 2008.

[17] V.Ramesh ， Dr.P.Subbaiah ， N. Koteswar Rao , M.Janardhana Raju , "Performance Comparison and Analysis of DSDV and AODV for MANET" , International Journal on Computer Science and Engineering, Vol. 02, No. 02, ISSN 0975-3397, pp. 183-188, India, 2010.

[18] S.S. Tyagi, R.K. Chauhan, "Performance Analysis of Proactive and Reactive Routing Protocols for Ad hoc Networks", International Journal of Computer Applications , Volume 1- No. 14, 2010.

[19] M.Saravana karthikeyan, K.Angayarkanni, Dr.S.Sujatha, "Throughput Enhancement in Scalable MANETs using Proactive and Reactive Routing Protocols ", Proceeding of International Multi-conference of engineers and computer scientists 2010, vol II, Hong Kong, March 2010

[20] Anuj K. Gupta, Member, IACSIT, Dr. Harsh Sadawarti, Dr. Anil K. Verma, "Performance analysis of AODV, DSR \& TORA Routing Protocols", International Journal of Engineering and Technology, Vol.2, No.2, ISSN: 17938236, April 2010.

[21] Prof. M.Neelakantappa, Dr.B.Satyanarayana, Dr. A.Damodharam, "Performance Improvement Techniques for Dynamic Source Routing Protocol in Mobile Ad Hoc Networks “, International Journal of Recent Trends in Engineering, , Academy Publisher, Vol 2, No. 2, India, November 2009.

[22] Ashgan Hussein Kamel, "A Framework for Integrated Routing Protocols for Mobile Ad Hoc Network", master thesis, Egypt, 2011. 